Acta Technologica Agriculturae 2

Nitra, Slovaca Universitas Agriculturae Nitriae, 2016, pp. 33-38

\title{
A REVIEW OF THE CHARACTERISTICS OF MODERN UNMANNED AERIAL VEHICLES
}

\author{
Georgi Valentinov HRISTOV*, Plamen Zlatkov ZAHARIEV, Ivan Hristov BELOEV \\ University of Ruse 'Angel Kanchev', Ruse, Bulgaria
}

\begin{abstract}
The main aim of this article is to present the modern unmanned aerial vehicles (UAVs) and the possibilities for real-time remote monitoring of flight parameters and payload data. In the introduction section of the paper we briefly present the characteristics of the UAVs and which are their major application areas. Later, the main parameters and the various data types for remote control and monitoring of the unmanned aerial vehicles are presented and discussed. The paper continues with the methods and the technologies for transmission of these parameters and then presents a general hardware model for data transmission and a software model of a communication system suitable for UAVs.
\end{abstract}

Keywords: unmanned aerial vehicles; telemetry; environmental monitoring; data transmission

Nowadays, the use of unmanned aerial vehicles (UAVs) for different tasks is quite popular, but before reaching such a level of acceptance this technology has been a subject of many changes in terms of both the methods for the management of aircrafts and their constructional characteristics (Keane and Carr, 2013). In the dawn of this technology, UAVs were mainly developed, constructed, operated and controlled by military organizations, with only few civil and commercial organizations being involved (Clark, 2000). Today, the majority of these devices are in service for commercial, civil or scientific organizations. Based on the purpose of mission, all modern UAVs can be operated in two modes - a manual or an autonomous flight mode. Back in the first days of UAVs, the situation was completely different an operator was required to perform all hovering, landing and take-off operations as well as to control the UAV during the entire flight (Erhard, 2000). Unlike their predecessors, modern UAVs are constructed using high-quality extremely light fibres and composite materials and are equipped with autonomous flight controller boards, which have allowed them to be successfully utilized for many different purposes, including road maintenance, town planning, monitoring, natural hazard warning, environmental studies and documentation of cultural heritage, surveillance and many more (Lei Ma et al., 2013).

\section{Construction and operation characteristics of UAVS}

From a construction point of view, the most used types of UAVs are fixed wing and multi-rotor aircrafts. Fixed wing UAVs are suitable for covering large areas of interest, while multi-rotor UAVs are much more suitable for smaller areas (Shlok et al., 2014). Multi-rotor UAVs, as their name suggests, are based on rotors and blades (Figure 1A), while fixed wing UAVs are similar in construction to airplanes (Figure 1B). Most UAVs are equipped with a camera or video recorder, which are used to acquire aerial images and videos.

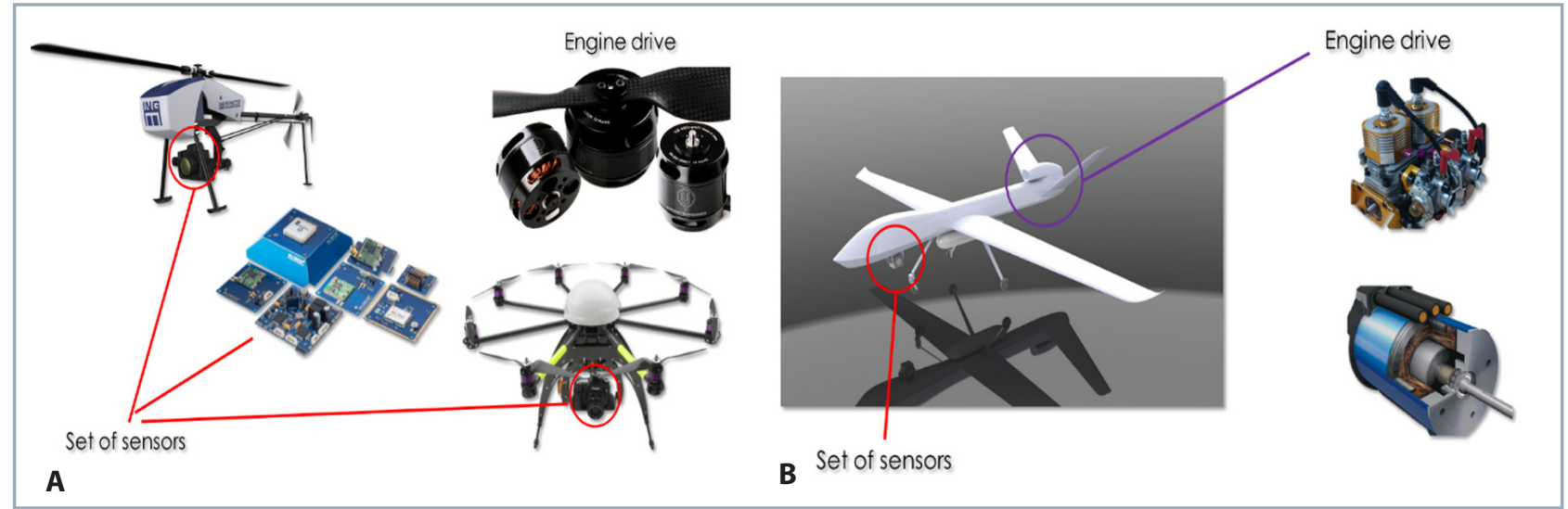

Figure 1 Multi-rotor (A) and fixed wing (B) UAVs

Source: Hristov, Zahariev and Beloev 


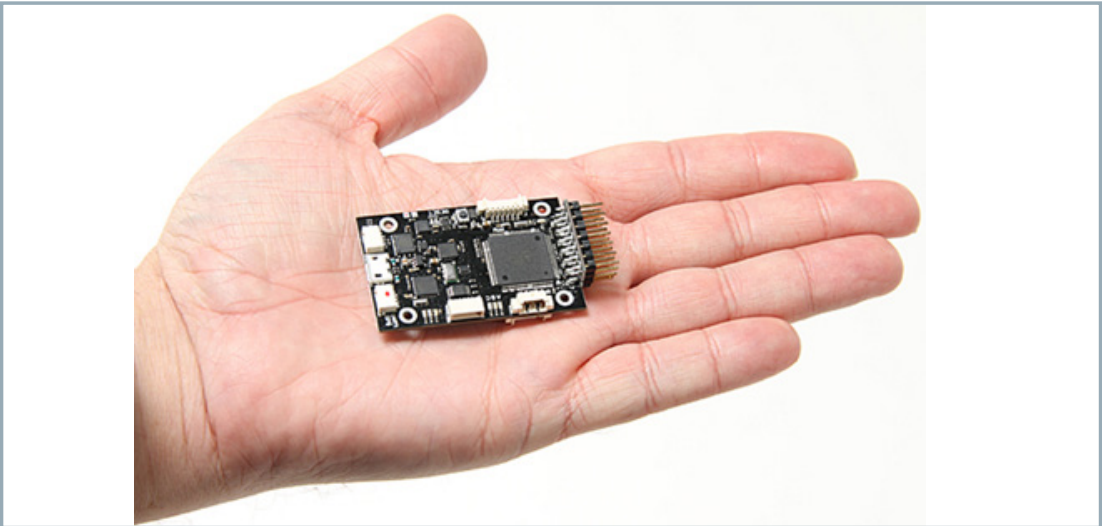

Figure 2 Advanced autopilot system

Source: Anderson, 2009

The cost of using an UAV, even if it requires the purchase of the vehicle, is much lower compared to the cost of using a manned aircraft solution or the use of ground teams with the corresponding equipment. An additional advantage of UAVs is their

capability to fly in almost any weather conditions. Other strong points of UAVs are their maintenance costs, their flexible manoeuvring capabilities, high resolution images they can produce, their ability to fly under the clouds, etc. UAVs are also easy to launch

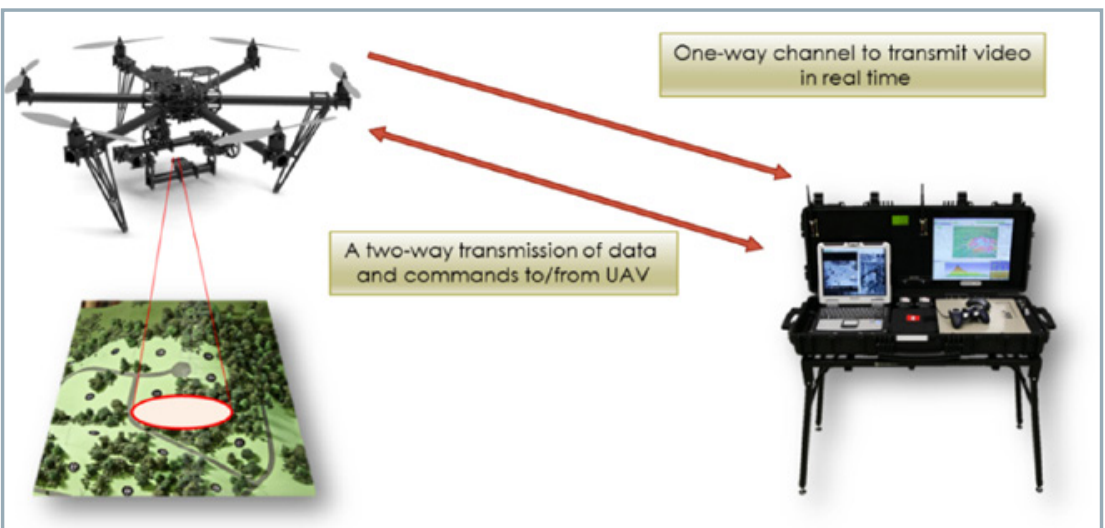

Figure 3 Communication between the UAV and the ground control station Source: Hristov, Zahariev and Beloev

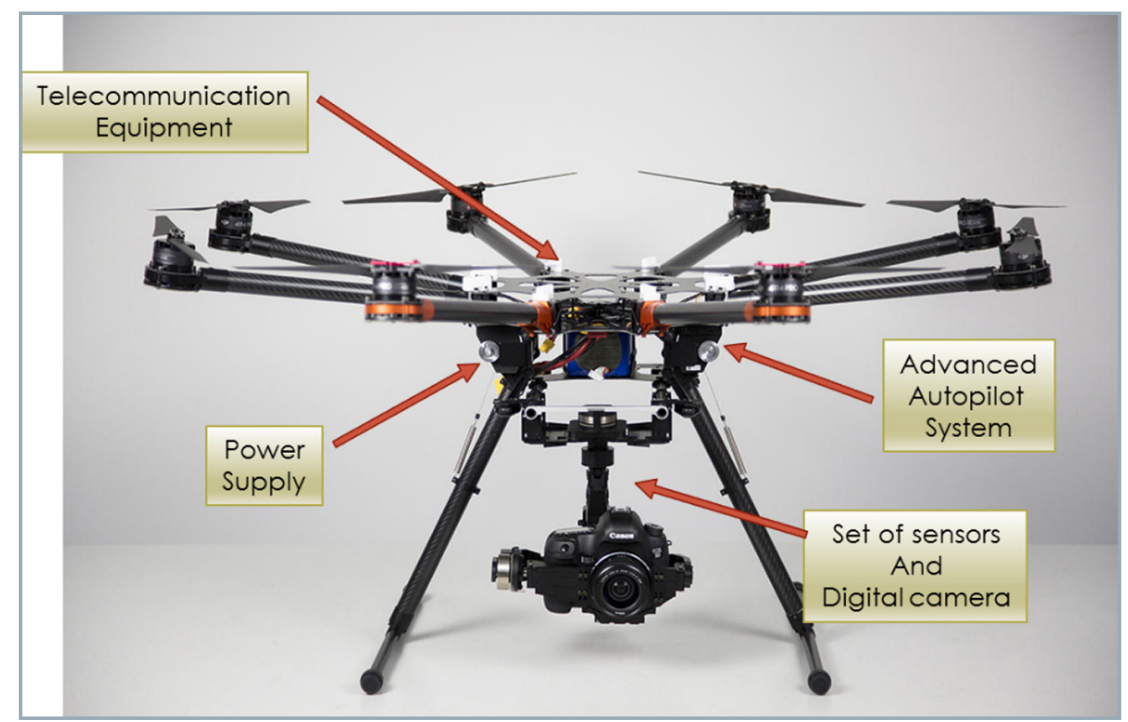

Figure 4 UAV equipped with the digital camera and set of sensors Source: Hristov, Zahariev and Beloev and land and are very safe to use. The disadvantages of these vehicles include their payload limitation and their small coverage, the large number of images that need to be taken and processed in order to obtain a highquality ortho-photo image and the large geometric distortion of produced images. In short, UAVs represent a low cost, time saving data collection solution, which is very easy to operate and maintain. One of the main reasons for this is a microcontroller, which is installed in UAVs and allows them to operate autonomously (Figure 2 ).

In order to set the UAV into the autonomous flight mode, the user or the operator of the vehicle needs to enter the initial position of the UAV and then record this information into the microcontroller (Hoffmann et al., 2007). The autonomous flight mode is usable for both fixed wing and multirotor UAVs. During the autonomous flight of the vehicle, the operator can monitor its flight parameters via a radio link. This link is established before the start of the mission and is between the UAV and a ground control station (Figure 3). The process of data collection using UAVs is possible even in cloudy weather conditions and the quality of images surpasses that of satellite images, which are taken by satellites located several hundred thousand kilometres above the surface.

Low altitude UAVs are extremely suitable for obtaining aerial photographs of small areas. This is usually done by installing a digital camera on the bottom of the UAV. Digital cameras are used primary because of the small size but high resolution images they can provide. Figure 4 shows an example of multirotor UAV with a digital camera attached underneath the fuselage of the vehicle.

The modern unmanned aerial vehicles have many on-board systems and can carry numerous cameras and sensing devices (Hristov and Zahariev, 2013). Nevertheless, without reliable communication between the UAVs and the ground station their mission is endangered of failure. There are several data types that play an essential role for UAVs and are used in communication processes with the control station (Dimc et al., 2006). These information types are flight control data, flight 
status data, payload data and payload control data. Every single one of the data types has its own requirements about the communications link, delay, bandwidth, transmission direction and few other communication parameters. One of the most important aspects of communication processes is the data link protection and the security and integrity of the above-mentioned data types. The communication exchange between the UAV and the base station is influenced by environmental conditions and above all it is subject to signal interception. The data exchange process and the data itself can be secured by complex protection features, like error robust coding techniques with error detection and correction capabilities, protocols with high error tolerance, systems for minimization of electromagnetic exposure to jammers, the use of directed or narrow beam antennas, frequency hopping methods, spread spectrum techniques and intelligent signal processing (Weinstein and Jones, 2003). Typical threats to data links are information interception, corruption and change. Countermeasures to these threats are the introduction of strict UAV data link access procedures and the implementation of authentication procedures between all communicating entities. A possible solution to some treats is also the use of highly secure cryptographic algorithms and data integrity protocols for encoding and protecting the structure of data.

\section{Flight control data}

The flight control data is transmitted from the ground station towards the UAV. It usually consists of packets, containing various commands for controlling the speed of the aircraft (Christopher et al., 2011). This is mainly accomplished by controlling the UAVs engines and motors, their rotations per minute, etc. Other flight control commands might include commands for changing the positions of elevators, the position of flaps, commands for extending or retracting the landing gears and other. All commands are sent from the ground control station (GCS) to the UAV trough a low speed radio uplink, which usually provides speeds of up to $30 \mathrm{Kbps}$. The flight control data is extremely time sensitive, but its volume is very low and thus presents no challenge for modern communication interfaces and standards. A very interesting fact is that this type of data is not mission critical. Even though it is always good to have means for the remote control of the UAV, in the cases when the uplink fails, the aircraft can still continue with its mission. This is possible when the UAV is capable of detecting the failed link and uses its autopilot feature. This feature requires more computational power and can drain the batteries of the UAV more quickly, but can also save the drone when the control uplink fails.

\section{Flight status data}

The flight status data (also known as telemetry data) is used for evaluating the parameters of the UAV. There are several categories of flight status data based on the observed parameters - UAV location data, battery condition data and spatial positioning data (Dimc et al., 2006). The UAV location data is used for presenting the global or the relative coordinates of the aircraft, as well as the distance to the home base. This is usually accomplished by reading and processing the data from the on-board GPS module. In the cases when there is no such component available, the received signal strength indicator (RSSI) can be used in combination with radio-based localization algorithms (like TDoA or AoA) to determine the relative position of the UAV. The battery condition data is used for determining the state of batteries and for estimation of possible flight time. Probably the most important flight status data types are the ones about the spatial position of the aircraft. They consist of information about the speed and the direction of the drone, its altitude and the vertical and horizontal offsets. Based on the values of received flight status data, the operator in the GCS can make modifications to the UAV flight plan, can monitor its mission progress or can even issue flight control instructions and commands, which are to be sent to the drone. Usually the flight status data is transmitted in a single direction downlink, which is capable of maintaining data rates of about $1 \mathrm{Mbps}$ or less. Similarly to the flight control data the flight status data is not mission critical, but provides means for more effective control of the drone.

\section{Payload data}

Payload data is the only mission critical data and thus the loss of the communication channel, which is used for its sending can cause the failure of mission tasks. The payload data can be defined as the data streams from various on-board sensor devices, including on-board cameras, temperature and pressure sensors and all other sensing devices. Due to the huge amount of data that has to be sent to the ground control station the payload data is usually sent using a separate radio link, which is a broadband high speed link with speeds of over 2 Mbps (Christopher et al., 2011). This link is both uplink and downlink. This is due to the nature of data, the requirements to acknowledge some of the sent information, the necessity to send additional data for maintenance of the sessions between the drone and the base station, etc. Sending and receiving the payload data still presents a lot of challenges, including how to format the data in a more appropriate way, how to maintain real-time transmission, how to provide high quality video data without overconsumption of the channel bandwidth and many other. One of the major problems concerning the transmission of video and audio data is the lack of suitable encoding standards. Currently the most widely used digital video encoding standards are the MPEG standards, but they are designed for low dynamics video sequences and thus are unsuitable for real time high-dynamic video from the on-board cameras of UAVs.

\section{Payload control data}

The payload control data is sent rarely and is even sometimes not transmitted at all. This type of data is used when the on-board camera has to be controlled [usually by Pan-Tilt-Zoom (PTZ) commands or some sort of camera control protocols], or when the on-board devices have to be switched on or off. The payload control data can be sent using the radio interface, used for the flight control and the flight status data, or using the less loaded uplink channel, which is used for acknowledging the payload data (Dimc et al., 2006). 
The lightweight UAV flight operations are restricted by its range or action radius and by the communication link capabilities. This is why it is very important to conduct a multi-factor analysis about the

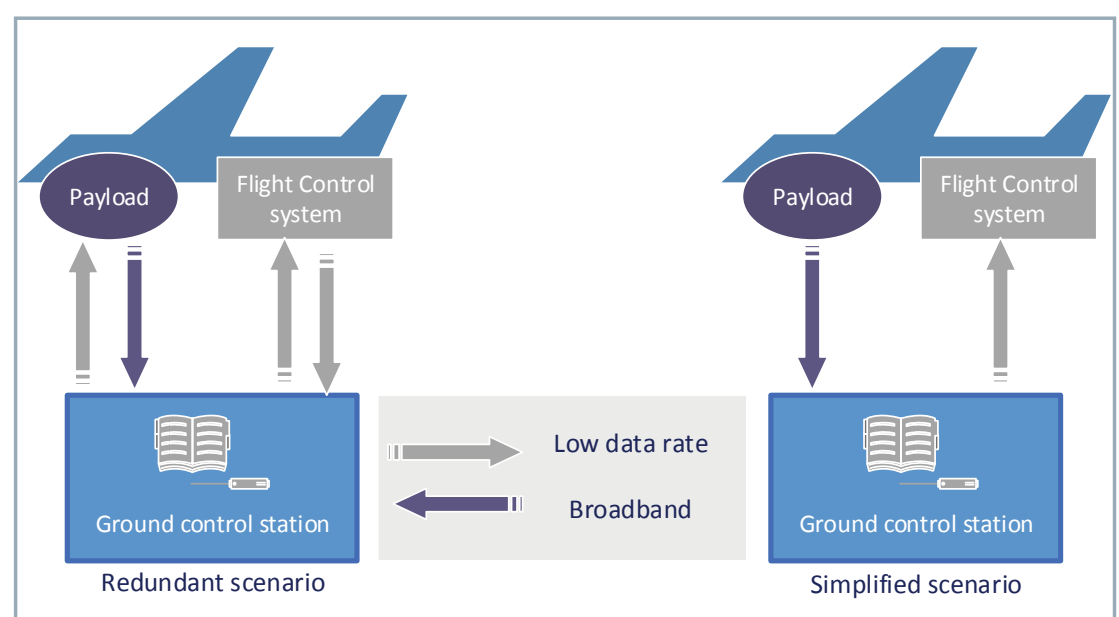

Figure 5 UAV data-link scenarios

Source: Hristov, Zahariev and Beloev

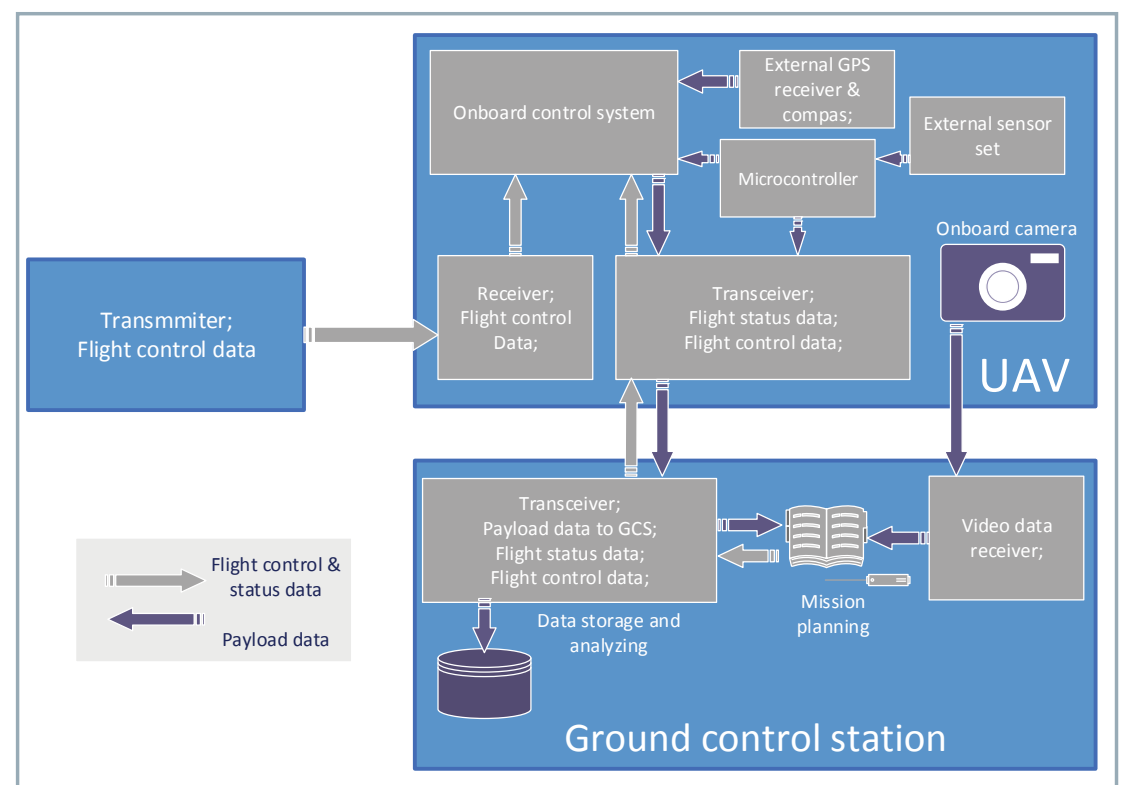

Figure 6 Conceptual design of UAV communication system Source: Hristov, Zahariev and Beloev possible balance between the set of mission vital requirements, UAV performance parameters and mission feasibility. This balance can be achieved by compromising between the volume of the data to be transmitted (defined requirements. The first model requires full redundancy of communication links, while the second one is aiming at the implementation of more simple communication platforms (Figure 5).

The UAV communication links requirements are defined by mission objectives and the flight environment. The UAV data-link connections, types and basics characteristics are summarized in Table 1 and presented in Figure 6.

The use of commercial off-theshelf communication systems defines not only communication frequencies but also the UAV transmitter power, its line-of-sight requirements, its communication range and the anticipated level of interference, which is something common for radio communication systems and depends on the frequency bands used. Clearly the communication between the UAV and its GCS influences the UAV system operational performances directly (Dimc et al., 2006; Christopher et al., 2011). The basic robustness of UAV communication links is secured by an additional on-board UAV power supply. Higher level of redundancy is ensured by the introduction of an additional radio link. With the multiplication of data links to and from the UAV and its GCS, i.e. with the multiplication of transceivers, receivers, transmitters and by the multiplication of communication frequencies, a continuous data link availability and sufficient communication exchange between the UAV and its GCS is guaranteed. The energy consumption

Table 1 Data-links of UAV system

\begin{tabular}{|l|c|c|c|}
\hline Data stream & Connection & Direction & Data rate \\
\hline Flight control & GCS to Autopilot & up & Low $<30 \mathrm{Kbps}$ \\
\hline Payload control & GCS to Payload & Low $<30 \mathrm{Kbps}$ & $1-2400 \mathrm{MHz}$ \\
\hline Flight status & Autopilot to GCS & down & High $<1 \mathrm{Mbps}$ \\
\hline Payload data & Payload to GCS & down & High $>1 \mathrm{Mbps}$ \\
\hline Traffic planning & GCS from to UAV & two way & Low $<30 \mathrm{Kbps}$ \\
\hline Mission planning & GCS from to UAV & two way & Low $<30 \mathrm{Kbps}$ \\
\hline
\end{tabular}

Source: Dimc et al., 2006 
for communication purposes is one of the major problems for UAVs. Using a redundant power supply is sometimes a solution, but it also requires space and increases the weight of the UAV - two of the mission critical parameters. Sometimes it is impossible to use a redundant power source. In those cases the communication approaches and the range of the UAV antennae array are the major elements for the success of the mission.

\section{Mission planning}

A mission planning software is commonly used to create the flight path for the mission of UAVs. Currently, there are many different mission planning software products and each of them is characterized by its own arrangement of virtual tools, by its capabilities and so on (Mission Planner Software; APM Planner 2.0; DJ Ground station). These products have many things in common, like the fact that all of them output flight data, spatial position of the aircraft and they all require certain input by the operator. All of the mission planning products need one mandatory parameter to be set before every autonomous flight - the starting point of flight path. These initial stating points are usually obtained using the global positioning system (GPS), and it is very common to use Google Earth or other mapping service to display the satellite images of the region for a better overview of the flight path and mission goals. Using the mission planning software the operator can set various control points, which the aircraft must follow, as well as other flight parameters - the altitude of the UAV in every stage of the flight, the speed of the vehicle, its flight pattern and so on. Based on these settings, the aircraft can perform area coverage flights, hovering tasks and so on. Once the flight plan is created using the mission planning software, the operator compiles the data into a specific file format and uploads the plan into the microcontroller of the UAV. One final step is required before the actual flight - the operator needs to check the motion sensors of the UAV and the corresponding control units - the elevator, the rudder, the motor, etc. Figure 7 shows the software, which can be used to plan and monitor the flight missions of fixed wing and multi-rotor UAVs.

An optional element for every UAV is the digital camera, which based on mission goals can be either mounted under the fuselage of the aircraft or inside it. These different mounting options allow the camera to be used either for $360^{\circ}$ coverage of the surroundings or for detailed aerial overview of the ground area. In the second case, the information about the flight plan and the resolution of photographs could be used for calculation of the precise area of ground coverage, which will be provided by a single image (Figure 8). The pixel size, the focal length and the flight altitude during image acquisition are important parameters and could be used for calculations of the number of objects in images, the length of an object in metric units and so on. All of the photographs, which are produced during the flight, could also be processed using a photogrammetric software, which would require additional data about the interior orientation, exterior orientation or a mean for aerial triangulation. Interior orientation involves complex calculation and comparison using clouds of points from different images and requires large database of photographs. Exterior orientation requires control points or tie point measurement. The location of ground control points or checkpoints could be manually obtained or provided by the real time kinematic global positioning system (RTKGPS).

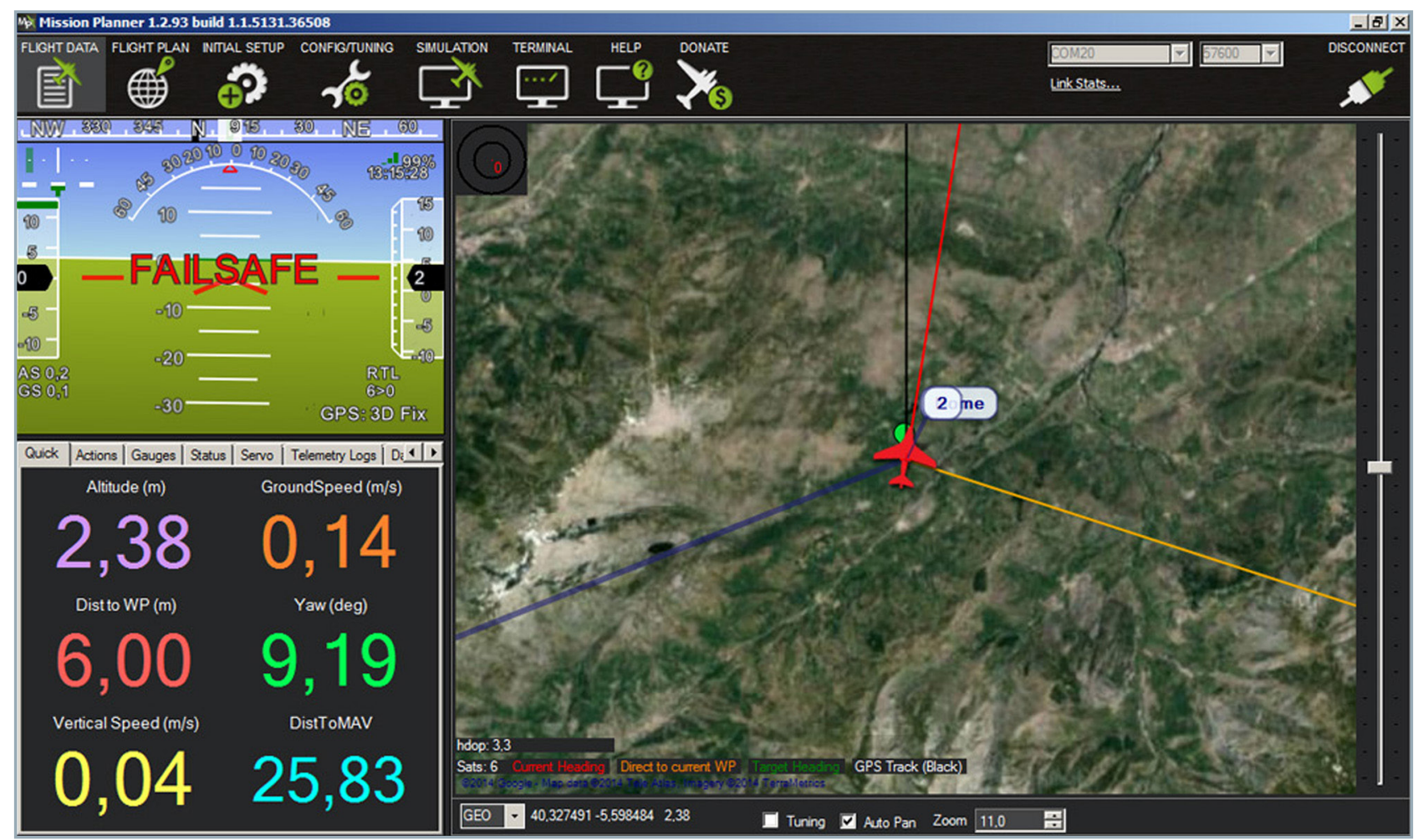

Figure 7 Graphical User Interface (GUI) software for mission planning (Mission Planner Software) 


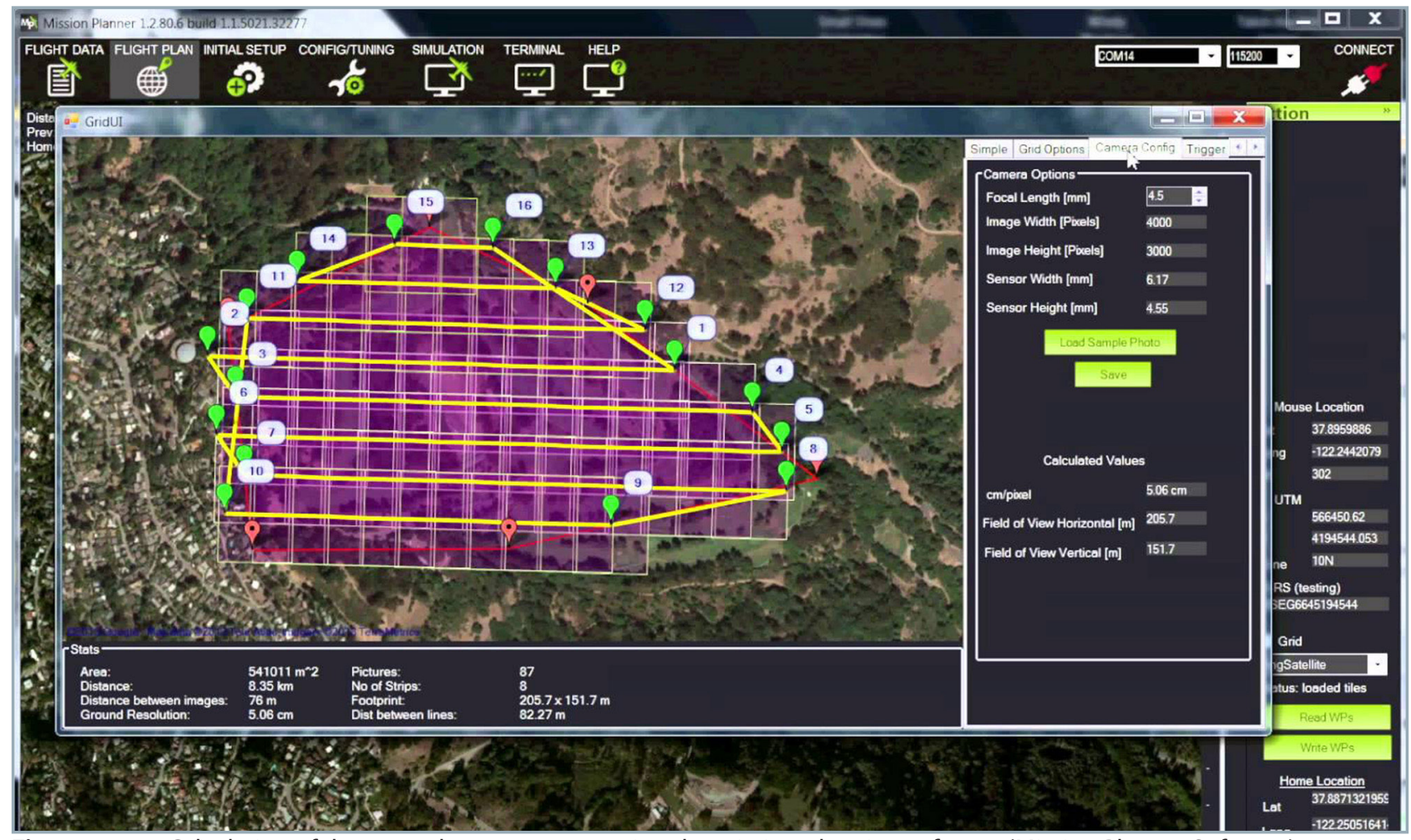

Figure 8 Calculation of the ground area coverage using the mission planning software (Mission Planner Software)

\section{Conclusion}

Over the years, UAVs have proven themselves as reliable and highly efficient tools for improving the performance and reducing the time and costs of many processes. Ever since their first flight, the potential of this technology was discovered, and many efforts were focused on providing better solutions for improving the performance of aircrafts. These efforts have led to the development of specialized miniature microcontrollers, high efficiency motors, advanced batteries and new airframes, which are constructed from sophisticated ultra-light composite materials. Using these advanced technologies, UAVs have been rediscovered as solutions to many issues and are continuing to improve our live and the way we explore our surroundings. Nevertheless, there are still many challenges in front of UAVs, like their relatively small payload capacity or their limited flight time. Besides these limitations, there are currently many legislative actions, which are aiming to define the rules for the use of UAVs and the areas where they will be allowed to fly. Despite these disadvantages, UAVs are considered as invaluable helpers for many tasks and in many application areas.

\section{References}

ANDERSON, C. 2009. DIY DRONES forum. Available at: https://3drobotics.com/

BARRETT, C. - BOSNIC, C. - PRIETO, A. - GOLEMBESKI, G. - BELL, D. 2011. UAV telemetry. In Multi-Disciplinary Senior Design Conference, Rochester, New York, 2011.

CARPENTER, M. - BONNEY, B. - DADE, S. 2011. APM Planner 2.0. Available at: https://3drobotics.com/
CLARK, R. M. 2000. Uninhabited combat aerial vehicles: Airpower by the people, for the people, but not with the people. CADRE Paper No. 8, Air University Press, Maxwell Air Force Base, Montgomery, AL, USA. DIMC, F. - MAGISTER, T. 2006. Mini UAV communication link systems. In $10^{\text {th }}$ International Conference on Traffic Sciences - ICTS 2006, Portoroz, Slovenia, 2006.

DJI Technology Co. Ltd DJI Ground station. Available at: http:// www.dji.com/products/ground-stations

ERHARD, T. P. 2000. Unmanned Aerial Vehicles in the United States Armed Services: A Comparative Study of Weapon System Innovation. PhD thesis, Johns Hopkins University, Baltimore, USA, 2000.

HOFFMANN, G. M. - HUANG, H. - WASLANDER, S. L. - TOMLIN, C. J. 2007. Quadrotor helicopter flight dynamics and control: Theory and experiment. In Proceeding of the AIAA Guidance, Navigation, and Control Conference, 2007.

HRISTOV, G. - ZAHARIEV, P. 2013. Approaches for remote monitoring of the parameters of an unmanned aerial vehicle. In Complex Control Systems, Institute of System Engineering and Robotics at the Bulgarian Academy of Sciences, vol. 12, 2013.

KEANE, J. F. - CARR S. S. 2013. A brief history of early unmanned aircraft. In Autonomous Systems, The Johns Hopkins University Applied Physics Laboratory, vol. 32, 2013, no. 3.

MA, L. - LI, M. - TONG, L. - WANG, Y. - CHENG, L. 2013. Using unmanned aerial vehicle for remote sensing application. In Proceedings of the $21^{\text {st }}$ International Conference on Geoinformatics (GEOINFORMATICS), Kaifeng, China, 2013.

OBORNE, M. 2007. Mission Planner Software. Available at: http:// planner.ardupilot.com/

SHLOK, A. - APOORVA, M. - KAMLESH, K. 2014. Design, construction and structure analysis of twinrotor UAV. In International Journal of Instrumentation and Control Systems (IJICS), vol. 4, 2014, no. 3, pp. 33-42. WEINSTEIN, E. - JONES, S. 2003. The differences between single antenna, antenna switching diversity, RF mixing diversity, true diversity and space-time diversity receivers. Avalon Systems Inc., AV010310/1-000 REV. X2 Dec. $3^{\text {th }} 2003$. 